\title{
Interaction of Baroreceptor and Chemoreceptor Reflex Control of Sympathetic Nerve Activity in Normal Humans
}

\author{
Virend K. Somers, Allyn L. Mark, and Francois M. Abboud \\ Departments of Internal Medicine and Physiology and Biophysics, Cardiovascular Center, University of Iowa, \\ and the Veterans Administration Medical Center, Iowa City, Iowa 55242
}

\begin{abstract}
Animal studies have demonstrated that activation of the baroreflex by increases in arterial pressure inhibits cardiovascular and ventilatory responses to activation of peripheral chemoreceptors (PC) with hypoxia. In this study, we examined the influences of baroreflex activation on the sympathetic response to stimulation of PC and central chemoreceptors in humans. PC were stimulated by hypoxia $\left(10 \% \mathrm{O}_{2} / 90 \% \mathrm{~N}_{2}\right)(n=6)$ and central chemoreceptors by hypercapnia $\left(7 \% \mathrm{CO}_{2} / 93 \% \mathrm{O}_{2}\right)(n=6)$. Responses to a cold pressor stimulus were also obtained as an internal reflex control to determine the selectivity of the interactive influence of baroreflex activation. Baroreflex activation was achieved by raising mean blood pressure by $>10 \mathrm{mmHg}$ with intravenous infusion of phenylephrine (PE). Sympathetic nerve activity (SNA) to muscle was recorded from a peroneal nerve (microneurography). During hypoxia alone, SNA increased from $255 \pm 92$ to $354 \pm 107 \mathrm{U} / \mathrm{min}(P<0.05)$. During $P E$ alone, mean blood pressure increased and SNA decreased to $87 \pm 45 \mathrm{U} / \mathrm{min}(P<0.05)$. With hypoxia during baroreflex activation with $P E$, SNA did not increase $(50 \pm 23 \mathrm{U} / \mathrm{min})$. During hypercapnia alone, SNA increased from $116 \pm 39$ to $234 \pm 72$ $\mathrm{U} / \mathrm{min}(\boldsymbol{P}<0.01)$. Hypercapnia during baroreflex activation with $P E$ increased SNA from $32 \pm 25 \mathrm{U} / \mathrm{min}$ during $P E$ alone to $61 \pm 26 \mathrm{U} / \mathrm{min}$ during hypercapnia and PE $(P<0.05)$. Like hypercapnia (but unlike hypoxia) the cold pressor test also increased SNA during PE. We conclude that baroreflex activation selectively abolishes the SNA response to hypoxia but not to hypercapnia or the cold pressor test. The inhibitory interaction of the baroreflex and the peripheral chemoreflex may be explained by convergence of baroreceptor and peripheral chemoreceptor afferents on neurons in the medulla. (J. Clin. Invest. 1991. 87:1953-1957.) Key words: hypoxia $\bullet$ hypercapnia • baroreflex $\bullet$ chemoreflex
\end{abstract}

\section{Introduction}

Baroreceptor and chemoreceptor reflexes exert considerable influence on autonomic control of the circulation, especially in situations involving stressors such as marked changes in blood pressure and in blood levels of oxygen and carbon dioxide. The individual contributions of baroreflexes and their responses to blood pressure alterations and of chemoreflexes and their responses to hypoxia and hypercapnia have been extensively studied in both animals $(1,2)$ and humans $(3,4)$. Thus far, however,

Address reprint requests to Dr. Francois M. Abboud, Department of Internal Medicine, University of Iowa College of Medicine, Iowa City, IA 52242

Received for publication 9 July 1990 and in revised form 14 December 1990.

The Journal of Clinical Investigation, Inc.

Volume 87, June 1991, 1953-1957 interactions of these reflexes have only been studied in animals. Heistad et al. have shown in dogs that baroreflex activation inhibits and deactivation augments the ventilatory response to stimulation of the peripheral chemoreceptors (5). In addition, Mancia et al. have demonstrated that vasoconstrictor responses to peripheral chemoreceptor stimulation are inhibited by elevation of blood pressure and activation of baroreceptor reflexes (6).

It has been difficult to study the interaction of baroreceptor and chemoreceptor reflexes in humans using measurements of vascular resistance and arterial pressure because the stimuli to these reflexes (vasoactive drugs and hypoxia) produce direct and confounding effects on the circulation. However, with the use of microneurographic recordings of sympathetic nerve activity (SNA), ${ }^{1}$ it is feasible to study the interaction of these reflexes in normal humans. We have shown that both hypoxia (peripheral chemoreceptor stimulation [7, 8]) and hypercapnia (primarily central chemoreceptor stimulation $[9,10])$ trigger increases in SNA in humans (4). Furthermore, baroreflex activation inhibits SNA. We, therefore, examined the effect of baroreflex activation (elevation of systemic pressure using intravenous phenylephrine infusions) on sympathetic nerve responses to stimulation of peripheral chemoreceptors (by hypoxia), to stimulation of central chemoreceptors (by hypercapnia), and to a cold pressor stimulus (used as an internal reflex control).

\section{Methods}

10 normal human volunteers ( 7 male, 3 female) aged $24 \pm 3$ yr were studied. All were nonsmokers and receiving no medication. Measurements were taken of heart rate (EKG), breathing patterns (pneumotach), blood pressure (Physio-Control Lifestat 200 semiautomated sphygmomanometer; Redmond, WA), $\mathrm{O}_{2}$ saturation (Nellcor $\mathrm{N}-1100$ $\mathrm{C}$ pulse oximeter, Hayward, $\mathrm{CA})$, end tidal $\mathrm{CO}_{2}$ (47210A capnometer; Hewlett-Packard Co., Andover, MA), central venous pressure, and sympathetic nerve activity to muscle using microneurography (11). SNA was measured directly by inserting a tungsten microelectrode into a nerve fascicle to muscle in the peroneal nerve. Sympathetic bursts were identified by inspection of the mean voltage neurogram and sympathetic activity was calculated as bursts/min $\times$ mean burst amplitude and expressed in arbitrary units (12). We also measured minute ventilation using a ventilation monitor (LS-75; Bourns, Riverside, CA).

Subjects were exposed to gas mixtures intended to induce either hypoxia $\left(10 \% \mathrm{O}_{2}, 90 \% \mathrm{~N}_{2}\right)$ or hypercapnia $\left(7 \% \mathrm{CO}_{2}, 93 \% \mathrm{O}_{2}\right)$. Subjects underwent measurement of baseline variables for $3 \mathrm{~min}$ while breathing room air. Then using a three-way valve, the subjects were exposed to either the hypoxic (six subjects) or hypercapnic (six subjects) stressors for $5 \mathrm{~min}$. Average values for the 5-min period of gas exposure were used in the comparisons. In one subject, responses to hypercapnia with and without phenylephrine (PE) were obtained over 3 min because of

1. Abbreviations used in this paper: $\mathrm{CPT}$, cold pressor test; CVP, central venous pressure; MBP, mean blood pressure; SNA, sympathetic nerve activity; $\dot{\mathrm{V}}_{\mathrm{E}}$, minute ventilation. 
inability to comfortably tolerate the stress for a longer period. Note that responses to hypoxia with and without PE were obtained in six subjects and to hypercapnia with and without PE in six subjects. Two subjects underwent both protocols (on separate days) and provided data for both the hypoxic as well as the hypercapnic stimuli. Exposure to each of the gas mixtures was performed in the baseline state and during elevation of systemic pressure using an intravenous infusion of PE. Infusion of PE was titrated to maintain mean blood pressure (MBP) at $\geq 10 \mathrm{mmHg}$ above baseline (thereby activating the baroreflexes). The interventions included: ( $a$ ) hypoxia or hypercapnia alone; $(b)$ PE alone; (c) hypoxia or hypercapnia during phenylephrine infusion. The order of the interventions was randomly allocated. At least $30 \mathrm{~min}$ separated the end of one intervention from the beginning of the next. Exposure to gas mixtures during the PE infusions was performed only after a constant infusion of $\mathrm{PE}$ for at least $10 \mathrm{~min}$ to ensure a steady state.

During hypoxia, hypocapnia secondary to the hyperventilation was avoided by titrating $\mathrm{CO}_{2}$ to maintain isocapnia.

We examined the effects of hypoxia and hypercapnia on SNA and compared these effects to those recorded when the baroreflexes were activated during the PE infusion. In four subjects, we further examined the effects of the cold pressor test (CPT) on SNA both in the baseline state as well as during the PE infusion. The CPT was performed by having subjects insert their hands to a predetermined depth into a container of ice for $2 \mathrm{~min}$.

The effects of hypoxia, hypercapnia, and the CPT during the baseline state were compared with their effects during baroreflex activation (by the PE infusion) using the Wilcoxon's signed rank test. To assess minute by minute changes in the measured variables, the data were further analyzed using a repeated measures one-way analysis of variance (Table III). Significance was assumed at the 5\% level. Values are expressed as mean \pm standard error.

The study was approved by the University of Iowa Human Subjects Review Committee, and each subject gave informed written consent.

\section{Results}

\section{(I) Effects of hypoxia (Table I)}

(a) On baseline variables. During hypoxia, $\mathrm{O}_{2}$ saturation fell from $99 \pm 0.4 \%$ to $83 \pm 1.4 \%(P<0.05)$, with an increase in minute ventilation $\left(\dot{\mathrm{V}}_{\mathrm{E}}\right)$ from $6.7 \pm 0.7$ to $11.8 \pm 1.0$ liters $/ \mathrm{min}(P$ $<0.05$ ) (Table III), and an increase in SNA from 255 \pm 92 to $354 \pm 107 \mathrm{U} / \mathrm{min}(P<0.05)$. MBP and central venous pressure (CVP) did not change.

(b) During phenylephrine. During PE alone, MBP increased by $\sim 10 \mathrm{mmHg}(P<0.05)$ and heart rate slowed by $\sim 11$ beats/min $(P<0.05)$. CVP increased from $0.7 \pm 0.5$ to

Table I. Comparison of Effects of Hypoxia Alone and during Phenylephrine Infusion

\begin{tabular}{|c|c|c|c|c|}
\hline & Baseline & Hypoxia & Phenylephrine & $\begin{array}{c}\text { Hypoxia } \\
+ \text { phenylephrine }\end{array}$ \\
\hline $\mathrm{O}_{2}$ Sat (\%) & $99 \pm 0.4$ & $83 \pm 1.4^{*}$ & $99 \pm 0.5$ & $84 \pm 1.8^{* \neq}$ \\
\hline $\mathrm{pCO}_{2}(\mathrm{mmHg})$ & $41 \pm 1.3$ & $40 \pm 1.3$ & $40 \pm 1.8$ & $41 \pm 1.4$ \\
\hline $\operatorname{MBP}(m m H g)$ & $80.0 \pm 3.6$ & $80.3 \pm 3.9$ & $89.7 \pm 2.3^{* 8}$ & $93.0 \pm 3.5^{* 5}$ \\
\hline HR (beats/min) & $65.8 \pm 2.4$ & $83.2 \pm 4.4^{*}$ & $54.5 \pm 3.1^{* \S}$ & $65.7 \pm 4.3^{8}$ \\
\hline $\mathrm{CVP}(\mathrm{mmHg})$ & $0.7 \pm 0.5$ & $0.3 \pm 1.0$ & $2.7 \pm 1.0$ & $3.3 \pm 0.9 *$ \\
\hline$\dot{\mathrm{V}}_{\mathbf{E}}$ (liters/min) & $6.7 \pm 0.7$ & $11.8 \pm 1.0^{*}$ & $7.1 \pm 0.8^{8}$ & $10.8 \pm 0.8^{* \pm}$ \\
\hline SNA $(U / \mathrm{min})$ & $255 \pm 92$ & $354 \pm 107^{*}$ & $87 \pm 45^{\S}$ & $50 \pm 23^{* 5}$ \\
\hline
\end{tabular}

$n=6 .{ }^{*} P<0.05$ compared to baseline; ${ }^{\ddagger} P<0.05$ compared to $\mathrm{PE}$ alone; ${ }^{8} P<0.05$ compared to hypoxia alone.

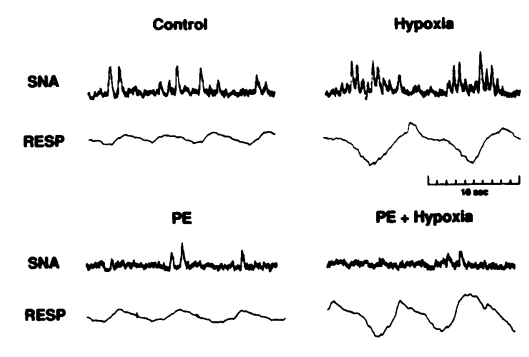

Figure 1. Recordings of sympathetic nerve activity $(S N A)$ and respiratory tracings (RESP) in a single subject, at baseline (control; top left), during hypoxia (top right), during $\mathrm{PE}$ infusion (bottom left), and during the combination of hypoxia and

$P E$ infusion. Expiration is indicated by upward movement of the respiratory trace and inspiration by downward movement. Note that hypoxia alone increased SNA, whereas PE suppressed SNA. Most importantly, hypoxia during PE resulted in a slight further suppression of SNA.

$2.7 \pm 1 \mathrm{mmHg}(P<0.05) . \dot{\mathrm{V}}_{\mathrm{E}}$ did not change, but SNA fell strikingly from $327 \pm 132$ to $87 \pm 45 \mathrm{U} / \mathrm{min}(P<0.05)$.

With hypoxia during PE, MBP was $93 \pm 3.5 \mathrm{mmHg}(\sim 13$ $\mathrm{mmHg}$ greater than during hypoxia alone) $(P<0.05)$. However, $\mathrm{O}_{2}$ saturation $(84 \pm 1.8 \%)$ and $\dot{\mathrm{V}}_{\mathrm{E}}(10.8 \pm 0.8$ liters $/ \mathrm{min})$ were similar to values during hypoxia alone (Table III). With addition of hypoxia during PE, SNA failed to increase and indeed tended to decrease even further than levels recorded during PE alone (87 \pm 45 to $50 \pm 23 \mathrm{U} / \mathrm{min}$ ) (Figs. 1 and 2 ).

\section{(II) Effects of hypercapnia (Table II)}

(a) On baseline variables. During hypercapnia, end tidal $\mathrm{CO}_{2}$ increased from $40 \pm 2.7$ to $53 \pm 1.5 \mathrm{mmHg}(P<0.05)$, with an increase in $\dot{\mathrm{V}}_{\mathrm{E}}$ from $6.7 \pm 0.6$ to $19.2 \pm 2.4$ liters $/ \mathrm{min}(P<0.05)$ (Table III) and an increase in SNA from $116 \pm 39$ to $234 \pm 72$ $\mathrm{U} / \mathrm{min}(P<0.05)$. MBP increased from $76.5 \pm 3.1$ to $82.3 \pm 3.1$ $\mathrm{mmHg}(P<0.05)$ and CVP increased from $0.8 \pm 0.6$ to $1.8 \pm 0.4$ $\mathrm{mmHg}(P<0.05)$.

(b) During phenylephrine. During PE alone, MBP increased to $84.7 \pm 2.3 \mathrm{mmHg}(P<0.05)$, heart rate slowed to $55.0 \pm 5.1$ beats $/ \mathrm{min}(P<0.05)$, and CVP increased to $3.2 \pm 0.6$
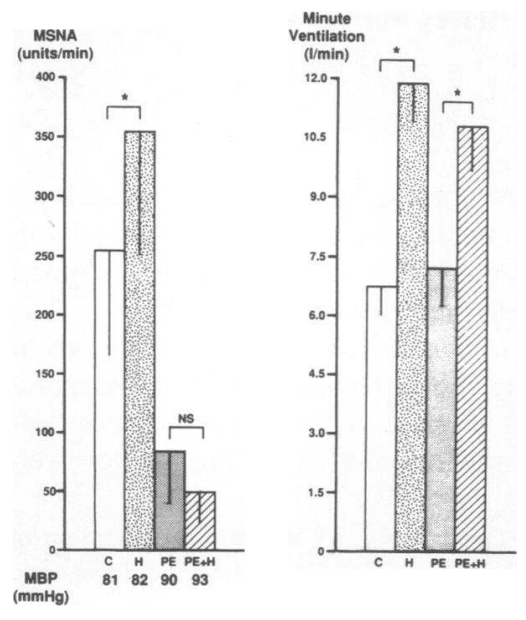

Figure 2. Group data for six subjects showing the effects of hypoxia $(H)$, phenylephrine alone $(P E)$, and hypoxia during $\mathrm{PE}(P E+H)$ on sympathetic nerve activity $(S N A$; left) and minute ventilation (right), as compared to measurements recorded in the control $(C)$ state. Note that hypoxia increased SNA and ventilation. PE markedly suppressed SNA. Hypoxia during PE resulted in no increase (and indeed a tendency to a

decrease) in SNA. Baroreflex stimulation by PE, however, did not significantly reduce the ventilatory response to hypoxia. Shown at the bottom of the left panel are values for MBP during each of the interventions. $\left({ }^{*} P<0.05\right)$. 
Table II. Comparison of Effects of Hypercapnia Alone and during Phenylephrine Infusion

\begin{tabular}{lcccc}
\hline & Baseline & Hypercapnia & Phenylephrine & $\begin{array}{c}\text { Hypercapnia } \\
\text { + phenylephrine }\end{array}$ \\
\hline $\mathrm{O}_{2}$ Sat (\%) & $99 \pm 0.4$ & $100 \pm 0.0$ & $99 \pm 0.4$ & $100 \pm 0.0$ \\
$\mathrm{pCO}_{2}(\mathrm{mmHg})$ & $40 \pm 2.7$ & $53 \pm 1.5^{*}$ & $41 \pm 1.4$ & $55 \pm 0.5^{* \neq}$ \\
$\mathrm{MBP}(\mathrm{mmHg})$ & $76.5 \pm 3.1$ & $82.3 \pm 3.1^{*}$ & $84.7 \pm 2.3^{*}$ & $93.7 \pm 1.7^{* \neq 5}$ \\
$\mathrm{HR}$ (beats/min) & $67.2 \pm 5.7$ & $71.2 \pm 6.5$ & $55.0 \pm 5.1^{*}$ & $61.3 \pm 4.7^{\S}$ \\
$\mathrm{CVP}(\mathrm{mmHg})$ & $0.8 \pm 0.4$ & $1.8 \pm 0.4^{*}$ & $3.2 \pm 0.6^{*}$ & $5.2 \pm 0.7^{* \neq}$ \\
$\dot{\mathrm{V}}_{\mathrm{E}}$ (liters/min) & $6.7 \pm 0.6$ & $19.2 \pm 2.4^{*}$ & $5.7 \pm 0.6$ & $20.2 \pm 1.8^{* \neq}$ \\
SNA (U/min) & $116 \pm 39$ & $234 \pm 72^{*}$ & $32 \pm 25^{*}$ & $61 \pm 24^{* \neq 5}$
\end{tabular}

$n=6 .{ }^{*} P<0.05$ compared to baseline; ${ }^{\ddagger} P<0.05$ compared to $\mathrm{PE}$ alone; ${ }^{8} P<0.05$ compared to hypercapnia alone.

mmHg $(P<0.05) . \dot{\mathrm{V}}_{\mathrm{E}}$ did not change. SNA fell to $32 \pm 25 \mathrm{U} /$ $\min (P<0.05)$.

With hypercapnia during $\mathrm{PE}, \mathrm{MBP}$ rose to $93.7 \pm 1.7$ $\mathrm{mmHg}(\sim 11 \mathrm{mmHg}$ greater than during hypercapnia alone) and CVP increased further to $5.2 \pm 0.7 \mathrm{mmHg}(P<0.05)$. Despite the increase in MBP and CVP with the addition of hypercapnia, SNA almost doubled (from $32 \pm 25$ to $61 \pm 24 \mathrm{U} / \mathrm{min} ; P$ $<0.05) . \dot{\mathrm{V}}_{\mathrm{E}}(20.2 \pm 1.8$ liters $/ \mathrm{min})$ and end tidal $\mathrm{CO}_{2}(55 \pm 0.5$ $\mathrm{mmHg}$ ) were similar to levels recorded during hypercapnia alone (Table III; Figs. 3 and 4).

\section{(III) Effects of the cold pressor test}

(a) On baseline variables. CPT increased MBP (from 77 \pm 1.9 to $90.5 \pm 5.9 ; P<0.05$ ) and SNA (from $162 \pm 40$ to $514 \pm 182 ; P$ $<0.05)$. CVP and $\dot{V}_{E}$ did not change significantly.

(b) During phenylephrine. CPT during PE resulted in a further increase in MBP from $91 \pm 3.2$ (PE alone) to $107 \pm 6.4$ $\mathrm{mmHg}(P<0.05)$. CVP was $5.2 \pm 0.5$ during CPT alone, $5.7 \pm 0.5$ during $P E$ alone, and $5.7 \pm 0.8$ with $C P T$ during $P E$. Despite the increase in MBP with CPT during PE, SNA increased from $5.5 \pm 1.9 \mathrm{U} / \mathrm{min}$ during $P E$ alone to $285 \pm 128 \mathrm{U} /$ min during CPT and PE (Fig. 5).

\section{Discussion}

The major new finding in this study was inhibition of the sympathetic nerve response to hypoxia (peripheral chemoreceptor stimulation) by baroreceptor activation in humans.

A strength of this study was the direct measurement of sympathetic nerve activity using microneurography. This enabled an analysis of these reflex interactions by providing a measure of the efferent sympathetic neural response to simultaneous activation of both baroreceptor and chemoreceptor afferents that is not influenced by direct effects of hypoxia, hypercapnia, and phenylephrine.

A limitation of the study was the fact that the use of phenylephrine to activate baroreceptors raises central venous pressure as well as systemic arterial pressure. Hence it is not possible to distinguish whether the interactions evident are due to activation of arterial baroreceptors or of cardiopulmonary receptors or both. In addition, we have not in this study examined the effects of baroreflex deactivation on the chemoreceptor responses.

A possible criticism is that the use of phenylephrine (an alpha agonist and therefore a vasoconstrictor) to activate the baroreflex may be associated with direct effects of phenylephrine (independent of the effects of the increased arterial pressure) on the chemoreceptors. If this were so, and vasoconstriction occurred in the chemoreceptors, we would expect an augmentation rather than an inhibition of the response to hypoxia, since vasoconstriction would increase the hypoxic stimulus. Furthermore, intravenous infusions of norepinephrine increase, rather than decrease the respiratory response to hypoxia in man (13). In any event, any effects of phenylephrine on the baroreflex itself is unlikely to have influenced our findings, since any such effect would be present during both the hypoxic and hypercapnic stresses. With regard to possible effects of phenylephrine on the baroreflexes, the doses of phenylephrine used in this study do not sensitize the arterial baroreceptors to graded levels of neck pressure (14).

We have confirmed earlier human studies demonstrating sympathetic neural activation by both hypoxia and hypercap-

Table III. Minute by Minute Changes in $\mathrm{O}_{2}$ Saturation, End Tidal $\mathrm{CO}_{2}$, and Ventilation during Hypoxia and Hypercapnia with and without Phenylephrine

\begin{tabular}{|c|c|c|c|c|c|c|}
\hline & Baseline & Minute 1 & Minute 2 & Minute 3 & Minute 4 & Minute 5 \\
\hline \multicolumn{7}{|l|}{ Hypoxia $(n=6)$} \\
\hline $\mathrm{O}_{2}$ Sat (\%) & $99 \pm 0.4$ & $89 \pm 1.9^{* *}$ & $85 \pm 1.0^{* \neq}$ & $83 \pm 1.3^{*}$ & $80 \pm 2.1^{*}$ & $78 \pm 2.4^{*}$ \\
\hline$\dot{\mathbf{V}}_{\mathbf{E}}($ liters/min $)$ & $6.7 \pm 0.7$ & $9.8 \pm 1.0^{* \neq}$ & $11.4 \pm 1.0^{* \neq}$ & $12.5 \pm 1.4^{*}$ & $13.3 \pm 1.1^{*}$ & $12.8 \pm 1.2^{*}$ \\
\hline \multicolumn{7}{|c|}{ Hypoxia + PE $(n=6)$} \\
\hline $\mathrm{O}_{2}$ Sat (\%) & $99 \pm 0.5$ & $92 \pm 1.5^{* \neq}$ & $84 \pm 1.4^{* \pm}$ & $82 \pm 2.5^{*}$ & $79 \pm 1.5^{*}$ & $77 \pm 2.5^{*}$ \\
\hline$\dot{\mathbf{V}}_{\mathbf{E}}$ (liters/min) & $7.1 \pm 0.9$ & $9.1 \pm 1.0^{* \neq}$ & $10.6 \pm 1.1^{* \neq}$ & $11.7 \pm 0.7^{*}$ & $11.8 \pm 0.6^{*}$ & $11.6 \pm 0.7^{*}$ \\
\hline \multicolumn{7}{|c|}{ Hypercapnia $(n=5)$} \\
\hline $\mathrm{pCO}_{2}(\mathrm{mmHg})$ & $42 \pm 0.8$ & $53 \pm 1.0^{* \pm}$ & $54 \pm 1.0^{*}$ & $55 \pm 1.1^{*}$ & $56 \pm 0.7^{*}$ & $56 \pm 0.7^{*}$ \\
\hline$\dot{\mathrm{V}}_{\mathrm{E}}$ (liters/min) & $6.3 \pm 0.6$ & $11.5 \pm 1.3^{* \pm}$ & $17.8 \pm 2.6^{* \ddagger}$ & $19.0 \pm 2.2^{*}$ & $24.3 \pm 3.3^{*}$ & $26.1 \pm 3.4^{*}$ \\
\hline \multicolumn{7}{|c|}{ Hypercapnia + PE $(n=5)$} \\
\hline $\mathrm{pCO}_{2}(\mathrm{mmHg})$ & $42 \pm 0.8$ & $53 \pm 1.0^{* \neq}$ & $54 \pm 1.0^{*}$ & $55 \pm 0.6^{*}$ & $56 \pm 0.5^{*}$ & $56 \pm 0.4$ \\
\hline$\dot{\mathbf{V}}_{\mathbf{E}}($ liters/min) & $6.7 \pm 0.7$ & $12.0 \pm 1.1^{* \neq}$ & $19.0 \pm 1.8^{* *}$ & $23.0 \pm 2.2^{*}$ & $25.4 \pm 2.6^{*}$ & $26.7 \pm 2.6^{*}$ \\
\hline
\end{tabular}

${ }^{*} P<0.05$ compared to baseline; ${ }^{\ddagger} P<0.05$ compared to the previous minute. $\mathrm{O}_{2}$ Sat, oxygen saturation. Note: The $\mathrm{O}_{2}$ saturation and ventilatory responses over time for hypoxia alone and hypoxia and phenylephrine were similar, as were the $\mathrm{pCO}_{2}$ and ventilatory responses over time for hypercapnia alone and hypercapnia and phenylephrine. 


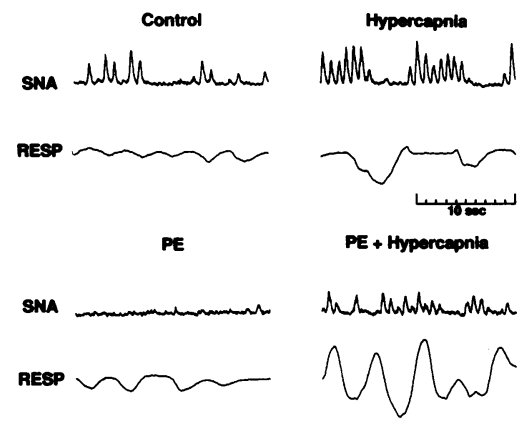

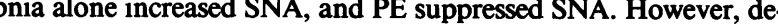
spite the suppression by PE, superimposed hypercapnia (unlike hypoxia) still elicited a substantial SNA response.

nia. More importantly, in this study we have shown in humans that baroreceptor activation inhibits the sympathetic responses to hypoxia. This baroreceptor-chemoreceptor interaction was previously described in animals $(5,6,15,16)$. In humans, this interaction appears to be specific for hypoxia, which activates primarily peripheral chemoreceptors. In contrast, during baroreflex activation, sympatho-excitation still occurs during hypercapnia, which stimulates primarily central chemoreceptors. Sympatho-excitation is also present in response to a potent nonspecific stimulus such as the cold pressor test. This specificity of the interaction between the baroreceptors and the peripheral (but not central) chemoreceptors is remarkably similar to an interaction that we reported earlier where ventilation (and thereby activation of thoracic afferents) inhibited the sympathetic response to hypoxia far more profoundly than it inhibited the sympathetic response to hypercapnia (4). These selective interactions may be explained by neurophysiological studies demonstrating that carotid baroreceptor and chemoreceptor neurons are distributed in close proximity in the solitary and paramedian reticular nuclei in the medulla, such that interneuronal connections might facilitate interactions between these reflexes (17).
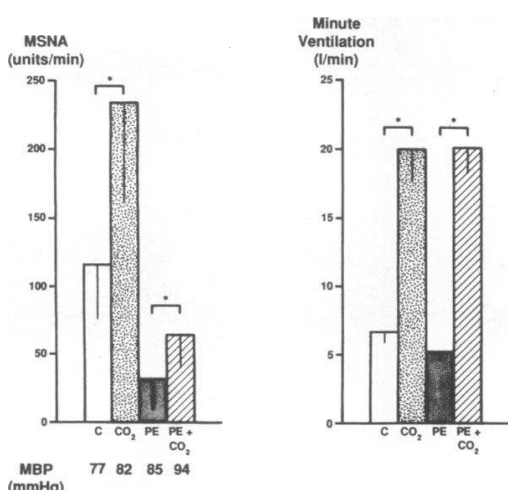

tion. Despite the marked suppression of SNA by PE, hypercapnia during PE still elicited an increase in SNA, unlike the complete suppression seen during hypoxia (Fig. 2). Baroreflex activation by PE did not influence the ventilatory response to hypercapnia. Values for MBP obtained during the various interventions are shown below the figure on the left.

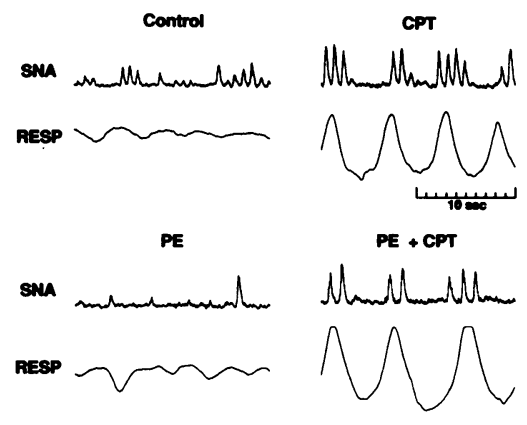

Figure 5. Recordings of sympathetic nerve activity $(S N A)$ and respiratory tracings (RESP) in a single subject, at baseline (control; top left), during the cold pressor test (CPT; top right), during a PE infusion (bottom left), and during the CPT imposed during the PE infusion. Baroreflex acti-

vation by PE did not abolish the SNA response to CPT (compare the response to hypoxia in Fig. 1).

An unexpected finding in this study was that SNA tended to decrease (not increase) when hypoxia was imposed during baroreceptor activation with phenylephrine. Activation of the baroreflex may explain the failure of SNA to rise when peripheral chemoreceptors are stimulated by hypoxia, but why would SNA tend to decrease? There are two possible explanations. First, when hypoxia was imposed during PE, there was a slight increase in blood pressure which would produce further baroreflex inhibition of SNA. Second, when hypoxia was imposed during PE, there was an increase in ventilation which could inhibit SNA by activating thoracic stretch receptors.

We did not see an inhibitory influence of baroreceptor activation on the ventilatory response to hypoxia, as has been reported in animals (5). This may be explained by the fact that the magnitude of the pressure change in animal studies $(>100$ $\mathrm{mmHg})$ far exceeded the pressure increase in this study $(\sim 10$ $\mathrm{mmHg}$ ). The significance of this absence of any ventilatory inhibition despite sympathetic inhibition is that the baroreflex chemoreflex interaction may more profoundly influence the sympathetic limb of the chemoreflex as compared to the ventilatory limb.

The results of this study shed light on our earlier report of sympathetic hyperresponsiveness to hypoxia in borderline hypertensives (18). We speculate that the baroreflex impairment known to occur in hypertension, may result in a loss of the inhibitory tonic or restraining influence of baroreceptors on the excitatory effect of chemoreceptors during hypoxia.

In conclusion, these data demonstrate a specific interaction between baroreceptors and peripheral chemoreceptors in regulation of sympathetic nerve activity in normal humans. Activation of baroreceptors by increases in arterial pressure with phenylephrine markedly inhibit the sympatho-excitatory response to stimulation of peripheral chemoreceptors with hypoxia. This inhibitory influence of baroreceptor activation was not observed during stimulation of central chemoreceptors with hypercapnia.

\section{Acknowledgments}

The authors wish to thank Mary Clary for expert technical assistance and Nancy Stamp for typing the manuscript.

The work was carried out with support of grants HL-24962 and HL-14388 from the National Heart, Lung, and Blood Institute of the National Institutes of Health. 


\section{References}

1. Calvelo, M. G., F. M. Abboud, D. R. Ballard, and W. Abdel-Sayed. 1970. Reflex vascular responses to stimulation of chemoreceptors with nicotine and cyanide: activation of adrenergic constriction in muscle and noncholinergic dilatation in dog's paw. Circ. Res. 27:259-276.

2. Walker, B. R., and B. L. Brizzee. 1990. Cardiovascular responses to hypoxia and hypercapnia in barodenervated rats. J. Appl. Physiol. 68:678-686.

3. Somers, V. K., A. L. Mark, D. C. Zavala, and F. M. Abboud. 1989. Influence of ventilation and hypocapnia on sympathetic nerve responses to hypoxia in normal humans. J. Appl. Physiol. 67:2095-2100.

4. Somers, V. K., A. L. Mark, D. C. Zavala, and F. M. Abboud. 1989. Contrasting effects of hypoxia and hypercapnia on ventilation and sympathetic activity in humans. J. Appl. Physiol. 67:2101-2106.

5. Heistad, D. D., F. M. Abboud, A. L. Mark, and P. G. Schmid. 1975. Effect of baroreceptor activity on ventilatory response to chemoreceptor stimulation. $J$. Appl. Physiol. 39:411-416.

6. Mancia, G. 1975. Influence of carotid baroreceptors on vascular responses to carotid chemoreceptor stimulation in the dog. Circ. Res. 36:270-276.

7. Wade, J. G., C. P. Larson, Jr., R. F. Hickey, W. K. Ehrenfeld, and J. W. Severinghaus. 1970. Effect of carotid endarterectomy on carotid chemoreceptor and baroreceptor function in man. N. Engl. J. Med. 282:823-829.

8. Lugliani, R., B. J. Whipp, C. Seard, and K. Wasserman. 1971. Effect of bilateral carotid-body resection on ventilatory control at rest and during exercise in man. N. Engl. J. Med., 285:1105-1111.

9. Korner, P. I. 1979. Handbook of Physiology. The Cardiovascular System The Heart. Sect. 2, Vol. I, Chap. 20. American Physiological Society, Bethesda, MD. 691-739.
10. Gelfand, R., and C. J. Lambertsen. 1973. Dynamic respiratory response to abrupt change of inspired $\mathrm{CO}_{2}$ at normal and high $\mathrm{PO}_{2}$. J. Appl. Physiol. 35:903913.

11. Vallbo, A. B., K.-E. Hagbarth, H. E. Torebjork, and B. G. Wallin. 1979. Somatosensory, proprioceptive, and sympathetic activity in human peripheral nerves. Physiol. Rev. 59:919-957.

12. Mark, A. L., R. G. Victor, C. Nerhed, and B. G. Wallin. 1985. Microneurographic studies of the mechanisms of sympathetic nerve responses to static exercise in humans. Circ. Res. 57:461-469.

13. Cunningham, D. J. C., E. N. Hey, J. M. Patrick, and B. B. Lloyd. 1963. The effect of noradrenaline infusion on the relation between pulmonary ventilation and the alveolar $\mathrm{PO}_{2}$ and $\mathrm{PCO}_{2} \mathrm{~s}$ in man. Ann. NY Acad. Sci. 109:756-770.

14. Ferguson, D. W., F. M. Abboud, and A. L. Mark. 1985. Relative contribution of aortic and carotid baroreflexes to heart rate control in man during steady state and dynamic increases in arterial pressure. J. Clin. Invest. 76:2265-2274.

15. Heistad, D. D., F. M. Abboud, A. L. Mark, and P. G. Schmid. 1974. Interaction of baroreceptor and chemoreceptor reflexes. Modulation of the chemoreceptor reflex by changes in baroreceptor activity. J. Clin. Invest. 53:12261236.

16. Mancia, G., J. T. Shepherd, and D. E. Donald. 1976. Interplay among carotid sinus, cardiopulmonary, and carotid body reflexes in dogs. Am. J. Physiol. 230:19-24.

17. Miura, M., and D. J. Reis. 1972. The role of the solitary and paramedian reticular nuclei in mediating cardiovascular reflex responses from carotid baroand chemoreceptors. J. Physiol. (Lond.). 223:525-548.

18. Somers, V. K., A. L. Mark, and F. M. Abboud. 1988. Potentiation of sympathetic nerve responses to hypoxia in borderline hypertensive subjects. $H y-$ pertension (Dallas). 11:608-612. 